Guest Editorial

\title{
Bulimia Nervosa: A Psychological Eating Disorder
}

Aneeza Tausesf ${ }^{1}$ and Sara Hayee ${ }^{2}$

${ }^{1}$ University of Faisalabad, Faisalabad, Pakistan

${ }^{2}$ Government Graduate College for Women, Samanabad, Lahore, Pakistan

Keywords:

Eating disorders, bulimia nervosa, psycological disorder

\section{How to Cite:}

Tausesf, A. ., \& Hayee, S. (2020). Bulimia Nervosa: A Psychological Eating Disorder: A Psychological Eating Disorder. DIET FACTOR (Journal of Nutritional \&Amp; Food Sciences), 1(01), 2-2. https://doi.org/10.54393/df.v1i01.7

\section{Corresponding author:}

Sara Hayee

Government Graduate College for

Women, Samanabad, Lahore, Pakistan

Sara_hayee1@hotmail.com

\section{Article History}

Received: $13^{\text {th }}$ January 2020

Accepted: $15^{\text {th }}$ February 2020

Published: $30^{\text {th }}$ June 2020
Eating disorders are multifaceted mental health problems which require medical treatments and counseling from a psychological expert. In extreme condition,these eating disorders can cause serious health problems and may be lethal if these are not diagnosed and treated timely. Eating Disorder Hope organization has conducted a survey which revealed that almost $\mathbf{4 0 \%}$ of teen age girls were suffering from at least one kind of eating disorder while $\mathbf{9 1 \%}$ of young females do dieting to reduce their weight. It has been estimated that about one million males and ten million females suffer from various types of eating disorders.

Bulimia nervosa is one of the eating disorders. It oftens develops in teenage or early adulthood. It is common in women than men. In bulimia nervosa, a person usually eats a lot in a short period of time. It seems that the person cannot control himself from eating food. It happens until they are painfully full and then they will purge to end their discomfort and to compensate their calories. They will purge by forced vomiting laxatives, diuretics or excessive exercise. Frequent self-induced vomiting can contribute to inflammation of parotid gland and can result in hand calluses. It is more common in families appearing in generations.

There are many risk factors for this disease like being overweight/obesity, socio-cultural pressures to have a well tonned body, psychological impacts resulting in stress and poor self-esteem. Dieting has become a part of our culture. There are many symptoms of bulimia. For example, patients live in a fear of gaining weight, they go through the repeated episodes of abnormal eating large amounts of food in single sitting. There is a feeling which develops in patients that they cannot control what they eat and what type of food they eat, and after bingering they are forcing themselves to vomit and do more exercise to stop weight gain.

If we leave bulimia untreated it will cause kidney problems, irregular heart rhythms, digestive issues and dental problems etc. In time treatment and diagnosis of bulimia helps the person to recover well and quick and the person can get rid of this disorder completely otherwise it can be life threatening. A person experiences bulimia to manage the emotional pain he is undergoing but it only worsens the condition and the response may be to ramp up obsessive thoughts and compulsive behaviors. Nearly half of bulimia patients have a co-occuring mood disorder and more than half of bulimia patients have co-occuring anxiety disorders. Self-harm is a common condition affecting $34 \%$ of those with bulimia. Recent studies on bulimia show that in 10-15\% of male members of our society who are living with this disorder, risk of suicide has increased. Statistics show that relapse of this disorder is very common. Relapse is occurring in about $\mathbf{3 0 - 5 0 \%}$ of the cases, and it is leading to common cause of death in suicide. For treatment of bulimia nervosa, you may need to go for several types. Bulimia can be reduced by taking anti-depressants or psychotherapy, but using both in combination is more fruitful to overcome this disorder. Psychotherapy is very important for patients, in which the person goes to the psychiatrist to discuss the issues related to bulimia. Educating the patient on nutrition also helps in this regard. A nutritionist can prescribe the food by which a person will feel the cravings for food to a lesser extent. In severe conditions, the patient can be admitted to a hospital because sometimes there is a need for treatment in a hospital. In many cases it is not possible to eradicate it completely. But before the situation goes wrong, we can guide a person to lead a healthier life with the help of professionally used treatments. Since we are living in an era where everyone is busy, it is important to guide and educate the children about 
a healthy life style. There is a need to give them confidence about their physical appearance, no matter what their weight, size and shape is. Enjoyable family meals should be arranged on daily basis or regularly. Talks on physical appearances, body weight and dieting should be discouraged at home. Exercise, walk, activities like sports and swimming can help young generation to adopt a healthy lifestyle and streamline their daily life. If you observe that someone in your friends or relatives is having issues related to food which is indicating that he or she can possibly has an eating disorder, you should talk to the person supportively and discuss the issue and help them in any way you can before the situation gets worse. 